\title{
Differentially expressed genes in Men1 knockout and wild-type embryoid bodies for pancreatic islet development
}

\author{
HONG-LI ZHANG, WEN-YI LI, CUI-PING ZHANG, YA-XIN ZHU, \\ LING WU, HONG-MEI LONG, GUO LI and MIN LUO
}

\begin{abstract}
Shanghai Institute of Endocrine and Metabolic Diseases, Shanghai Clinical Center for Endocrine and Metabolic Diseases, Department of Endocrinology, Ruijin Hospital, Shanghai Jiaotong University School of Medicine, Shanghai 200025, P.R. China
\end{abstract}

Received September 7,2010; Accepted December 20, 2010

DOI: $10.3892 / \mathrm{mmr} .2011 .409$

\begin{abstract}
The Men1 gene has been identified as the gene responsible for MEN-1, a hereditary syndrome transmitted as an autosomal dominant trait. Disruption of the Men1 gene results in defects in the development of multiple organs, including pancreatic islets. Homozygous disruption of Men1 in mice causes embryonic lethality, making it difficult to determine the genes involved in defects of pancreatic islets during embryonic development. In this study, embryoid bodies formed from null mutant $\left(\mathrm{Men1}^{-} /\right)$and wild-type (Men1 $\left.{ }^{+/+}\right)$embryonic stem cells were used as a model system to investigate the effects of the Men1 gene on pancreatic islet development. Using RT-PCR, SOX17, FOXA2 and NKX2.2 were found to be differentially expressed between the two embryoid bodies. Additionally, the gene expression profile of these Men1\% embryoid bodies was characterized in detail by DNA microarray techniques, and a series of putative menin-targeted genes was identified. Our study suggests a critical role for Men1 in pancreatic islet development, and indicates that genes such as SOX17, FOXA2, NKX2.2 and SOX4 are potential targets of Men1.
\end{abstract}

\section{Introduction}

The Men1 gene has been identified as the gene responsible for MEN-1, a hereditary syndrome transmitted as an autosomal dominant trait. The disease is characterized by a predisposition to multiple endocrine tumors in the parathyroid, endocrine pancreas and anterior pituitary, as well as, though less frequently, adrenal cortical tumors, and lastly foregut diffused endocrine carcinoids $(1,2)$. MEN-1 syndrome is closely linked to the development of pancreatic endocrine tumors, and forms the basis for the classification of these tumors

Correspondence to: Dr Guo Li, Shanghai Institute of Endocrine and Metabolic Diseases, Shanghai Clinical Center for Endocrine and Metabolic Diseases, Department of Endocrinology, Ruijin Hospital, Shanghai Jiaotong University Medical School, 197 Ruijin 2nd Road, 200025, Shanghai, P.R. China

E-mail: liguorj@sohu.com

Key words: Men1, embryoid body, pancreatic islet, development into sporadic and familial variants. Among MEN-1 patients, 30-50\% develop symptomatic pancreatic endocrine tumors, while $100 \%$ of patients have been found to harbor small nonfunctioning pancreatic endocrine tumors in pathology studies (3). Similar to humans, Men1 knockout mice develop multiple endocrine tumors, mainly in the pancreas, parathyroid, pituitary glands and adrenal glands, with insulinomas being the most common lesions. In a study by Bertolino et al, more than $80 \%$ of pancreatic $\beta$-cell-specific ablation mice developed multiple pancreatic islet adenomas by 60 weeks of age, with islet lesions exhibiting features of multistage tumor progression, including $\beta$-cell dedifferentiation and angiogenesis (4). Taken together, the evidence suggests that the Men1 gene, as a classic tumor suppressor, is involved in the development of pancreatic islet tumors. It is generally accepted that abnormalities in development and differentiation may lead to tumorigenesis. Thus, the Men1 gene may play a crucial role in the development and differentiation of pancreatic islets.

The protein product of the Men1 gene, termed menin, is ubiquitously expressed and targeted to the nucleus, and has been reported to interact with a variety of proteins, including JUND, SMAD3, PEM, NM23, NF-кB and RPA2 (5-10). In mice with disrupted Men1, menin was found to be required for the development of several organs and tissues, including the neural tube and liver (11). Moreover, a recent report suggested that menin is involved in the development of pancreatic endocrine cells; the authors found that menin is required for the formation of NEUROG3-expressing cells during the crucial step of the second pancreatic transition, in which progenitor cells commit to endocrine differentiation (12). However, homozygous Men1 knockout mice die in utero between embryonic days 11.5 and 12.5 (13). This lethality has made it difficult to study changes in gene expression in the pancreatic islets of Men $1 \%$ mouse, and the genes affected by menin in the embryonic development of endocrine cells remain to be identified.

In the present study, we used embryoid bodies formed from wild-type $\left(\mathrm{Men1}^{+/+}\right)$and Men1 null mutant (Men1\%) embryonic stem (ES) cells as a model to investigate the physiological function of menin in pancreatic islet development. Differences in the morphology of the Men1\% and Men1+/+ embryoid bodies were first examined, then the expression profiles of two different categories of genes relevant to pancreatic islet development and differentiation, including 
Table I. List of gene-specific primers.

\begin{tabular}{lll}
\hline Gene & \multicolumn{1}{c}{ Forward primer } & \multicolumn{1}{c}{ Reverse primer } \\
\hline SOX17 & CTCGGGGATGTAAAGGTGAA & ACGAGAGCGTGCAGGAAG \\
GSC & GCCTGGGCTACAACAGCTAC & CGTCTGGGTACTTCGTCTCC \\
FOXA2 & CCCGGGACTTAACTGTAACG & GCGCCCACATAGGATGAC \\
NKX6.1 & TACTTGGCAGGACCAGAGAG & CGCTGGATTTGTCTTTTC \\
NKX2 2 & AAAGGTATGGAGGTGACGCCT & AGCTGTACTGGGCGTTGTACT \\
FGF2 & AGCGGCTCTACTGCAAGAAC & CCGTTTTGGATCCGAGTTTA \\
HNF-6 & AAGCCCTGGAGCAAACTC & CTGGGAGATGGTGATTTGTAA \\
PDX1 & TGTAGGCAGTACGGGTCCTC & CCACCCCAGTTACAAGCTC \\
IGF1 & ACACTGACATGCCCAAGACT & GTTTCGATGTTTGCAGGTT \\
HNF4A & ACACGTCCCCATCTGAAG & CTTCCTTCTTCATGCCAG \\
SOX4 & CTCTCCTCTCCTGCCTCTTG & GGCAGTTTCAGCTCCTCATC \\
BTC & AGATGGGAACACAACCAGAACAC & AACAGGTCCACTCGCTCACAC \\
FOXA1 & AAGCCCGTGTTGGCGTAG & CAGGGTTGGATGGTTGTGTC \\
IGF2 & AAGCCCGTGTTGGCGTAG & CAGGGTTGGATGGTTGTGTC \\
GAPDH & AACGACCCCTTCATTGAC & TCCACGACATACTCAGCAC \\
\hline
\end{tabular}

PDX1, HNF6, IGF1, NKX6.1, NKX2.2, HNF4 $\alpha$, FGF2 and HNF6, were determined. The gene expression profile of genes involved in endoderm development (SOX17, GSC and FOXA2) was examined in cultured Men1\% embryoid bodies, and characterized in detail by microarray techniques.

\section{Materials and methods}

Culture of embryonic stem cells and formation of embryoid bodies. Men $1^{-}$and $\mathrm{Men}^{+/+}$ES cells, kindly provided by Dr Changxian Zhang (Université Claude Bernard Lyon 1), were maintained in an undifferentiated state on a feeder layer of Mitomycin C (Sigma-Aldrich, St. Louis, MO, USA) -treated mouse embryonic fibroblasts in knockout DMEM (Gibco, CA, USA) supplemented with $15 \%$ ES cell qualified fetal bovine serum (Gibco), 1\% nonessential amino acids (Gibco), 2 mmol/1 L-glutamine (Sigma-Aldrich), $0.1 \mathrm{mmol} / 1 \beta$-mercaptoethanol (Sigma-Aldrich), $100 \mathrm{U} / \mathrm{ml}$ penicillin, $100 \mu \mathrm{g} / \mathrm{ml}$ streptomycin (Sigma-Aldrich) and 1,500 U/ml leukocyte inhibitory factor (Chemicon, USA) at $37^{\circ} \mathrm{C}$ in $5 \% \mathrm{CO}_{2}$. To initiate differentiation, the ES cells were removed from the feeder layers with $0.25 \%$ Trypsin plus $0.05 \%$ EDTA and plated on 6-well non-adherent sterile dishes at a density of $2 \times 10^{5}$ cells/well in the medium described above in the absence of leukocyte inhibitory factor. The embryoid bodies were cultured for 9 days, with the culture medium changed every 2 days, and were collected for morphological examination under a phase contrast microscope every day.

RT-PCR for gene expression patterns. Total RNA was isolated from the Men $1 \%$ and Men $1^{+/+}$embryoid bodies using the RNeasy Mini kit (Qiagen, Germany), and the isolated RNA was treated by RNase-free DNase I to eliminate any residual genomic DNA. First-strand cDNA synthesis from $5 \mu \mathrm{g}$ total RNA was performed using Superscript II (Invitrogen, USA) and random hexamer primers in a $20 \mu \mathrm{l}$ reaction according to the manufacturer's instructions. PCR reaction components were purchased from Qiagen. Whenever possible, intron-spanning primers were designed from published mouse sequences (EMBL) and a BLAST search was carried out against the nucleotide database to verify their specificity. The primer sequences are listed in Table I. PCR was performed using $1 \mu \mathrm{l}$ of the first-strand DNA preparation and $10 \mu \mathrm{mol} / 1$ of each primer under the following conditions: denaturation at $95^{\circ} \mathrm{C}$ for $1 \mathrm{~min}$, annealing at $55-60^{\circ} \mathrm{C}$ for $1 \mathrm{~min}$, and elongation at $72^{\circ} \mathrm{C}$ for $1 \mathrm{~min}$ for 30 or 35 cycles, followed by extension at $72^{\circ} \mathrm{C}$ for $10 \mathrm{~min}$. The products were analyzed on $1.2 \%$ agarose gel and images were captured with a UV-sensitive camera. Three independent experiments were carried out from three different ES cell aliquots, with reproducible results.

Quantitative PCR analysis. Quantitative PCR amplification and detection were performed with SYBR Premix Ex Taq Mixes (Takara, Japan) according to the manufacturer's protocol on an ABI PRISM 7000 Sequence Detection System (Applied Biosystems, Foster City, CA, USA). The specific primer sequences are listed in Table I. Cycle parameters were $95^{\circ} \mathrm{C}$ for $15 \mathrm{~min}$, then 40 cycles at $95^{\circ} \mathrm{C}$ for $15 \mathrm{sec}$ and $60^{\circ} \mathrm{C}$ for $1 \mathrm{~min}$. As a control, the mRNA level of GAPDH was determined in the real-time PCR assay for each RNA sample and was used to correct experimental variations. Melting curves were performed using Dissociation Curves software (Applied Biosystems) to ensure that only a single product was amplified. Quantification was performed in duplicate, and the experiments were repeated independently three times. Gene expression was normalized to GAPDH levels.

Microarray analysis. Total RNA from the Men1 $\%$ and Men1 $1^{+/+}$ embryoid bodies was extracted using the RNeasy Mini kit (Qiagen) according to the manufacturer's instructions. The differential expression of genes in the Men1\% and $\mathrm{Men}^{+/+}$ embryoid bodies was examined by the microarray technique according to the Affymetrix microchip array protocol (Affymetrix, Santa Clara, CA, USA). These samples were 


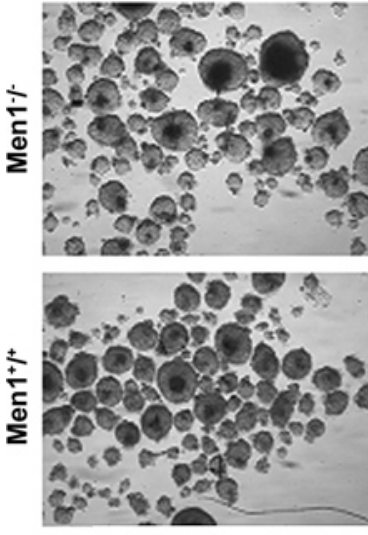

Day 3

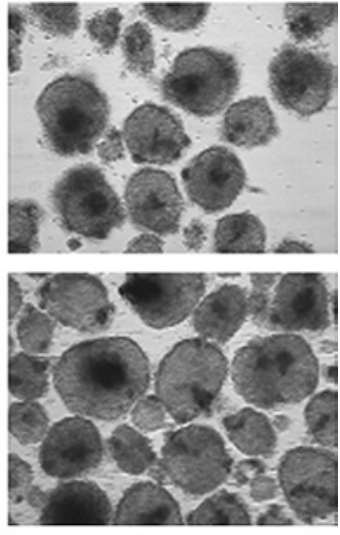

Day 5
Figure 1. Phase contrast microscopy of Men1\% and Men1 $1^{+/+}$embryoid bodies formed from ES cells. The size and number of embryoid bodies formed from Men $1 \%$ ES cells were similar to those formed by $\mathrm{Men}^{+/+}$ES cells on days 3 and 5. Magnification, x50 (day 3); x100 (day 5).

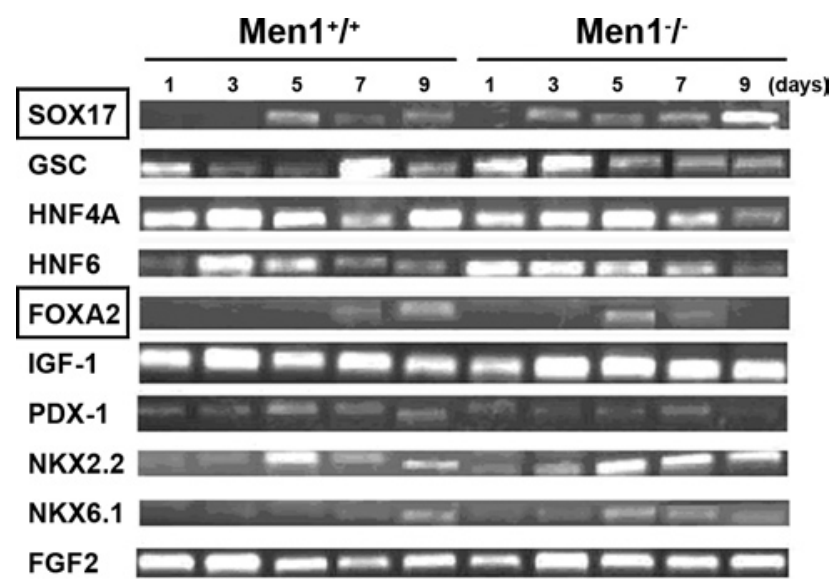

Figure 2. Gene expression in $\mathrm{Men}^{\%} \%$ and $\mathrm{Men} 1^{+/+}$embryoid bodies formed from ES cells. In Men1\% embryoid bodies, SOX17 expression was detected on day 3, while in $\mathrm{Men}^{+/+}$embryoid bodies it was not observed until day 5 . FOXA2 expression was detected on day 5 in Men $1 \%$ embryoid bodies, but on day 7 in $\mathrm{Men}^{+/+}$embryoid bodies. There were no differences between the expression patterns of GSC, PDX1, IGF1, FGF2, HNF6, NKX6.1, NKX2.2 and $\mathrm{HNF} 4 \alpha$.

subjected to cDNA array analysis using the Mouse430_2.0 microarray (Affymetrix), which contains 45,000 probe sets and analyzes the expression level of over 39,000 transcripts and variants from more than 34,000 well-characterized mouse genes. Microarray results were analyzed and scatter plots were generated using Microarray Suite 5.0 software (Affymetrix). Data analysis was performed using GeneSpring 6.2 software (Silicon Genetics, Redwood City, CA, USA).

\section{Results}

Morphology of Men1 null mutant and wild-type embryoid bodies. To study the differentiation capacity of Men $1 \%$ ES cells, an embryoid body formation assay was performed, which recapitulated specific features of early embryogenesis in vitro. Morphological analysis showed that embryoid bodies formed
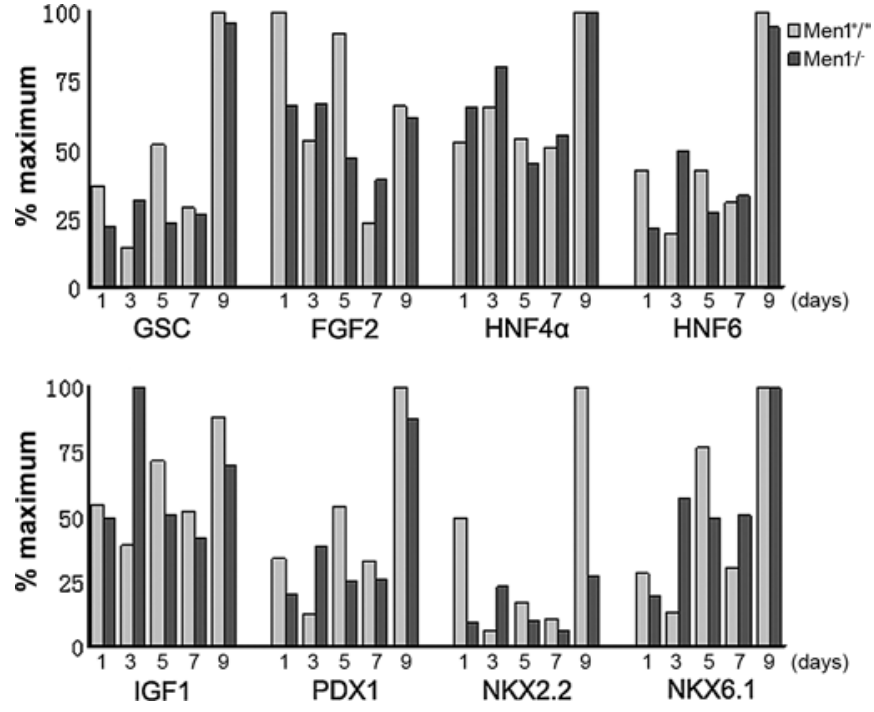

Figure 3. Expression profile of several genes in the two embryoid bodies as determined by real-time RT-PCR. Gene expression was normalized to GAPDH (average of two samples).

by Men1\% ES cells were similiar in size to those formed by Men $1^{+/+}$ES cells during the 9 days of suspension culture. The data suggest that the proliferation and differentiation capacity of ES cells was not impaired in the absence of menin, at least not in terms of morphology (Fig. 1).

Gene expression pattern determined by $R T-P C R$. As an initial approach to evaluate definitive endoderm development and the pancreatic islet development process in embryoid bodies, the expression of the FOXA2, SOX17, GSC, PDX1, IGF1, HNF6, FGF2, NKX6.1, NKX2.2 and HNF4 $\alpha$ genes was determined by RT-PCR. Among these genes, a difference between the gene expression patterns in the Men1\% and Men1 $1^{+/+}$embryoid bodies was observed for SOX17 and FOXA2. SOX17 expression in the Men1\% embryoid bodies was detected on day 3, while in $\mathrm{Men1}^{+/+}$embryoid bodies it was not observed until day 5. The expression pattern of FOXA2 was similar to that of SOX17, with its expression detected on day 5 in the Men1\% embryoid bodies and on day 7 in the Men $1^{+/+}$embryoid bodies. Since SOX17 and FOXA2 are markers of definitive endoderm development, these findings indicate that Men1\% ES cells may preferentially initiate differentiation towards the endodermal pathway. No differences between the time of gene expression of any of the other genes were observed (Fig. 2).

Gene expression profiles determined by real-time PCR. Genes differentially expressed between the Men1\% and Men1+/+ embryoid bodies were further quantitatively investigated using real-time PCR. The expression levels of GSC, PDX1, IGF1, FGF2, IGF1, NKX6.1, NKX2.2 and HNF4 $\alpha$ differed between the two embryoid body models during cultivation, with the most marked difference observable on days 3-5 Subsequently, expression levels tended to be similar, with the exception of NKX2.2, which was down-regulated at day 9 in the Men1\% embryoid bodies. HNF4 $\alpha$ was the only exception, showing no significant difference between the two embryoid bodies during the cultivation period (Fig. 3). 
Table II. Comparison of qPCR with microarray results.

\begin{tabular}{|c|c|c|c|c|c|}
\hline \multirow[t]{2}{*}{ Accession no. } & \multirow[t]{2}{*}{ Name } & \multicolumn{2}{|c|}{ Microarray } & \multicolumn{2}{|c|}{ qPCR } \\
\hline & & \multicolumn{2}{|c|}{ Fold change } & \multicolumn{2}{|c|}{ Fold change ${ }^{a}$} \\
\hline NM_009238 & SOX4 & $\downarrow$ & 2.30 & $\downarrow$ & 1.71 \\
\hline NM_007568 & BTC & $\downarrow$ & 24.25 & $\downarrow$ & 1.55 \\
\hline NM_008259 & FOXA1 & $\downarrow$ & 2.46 & $\downarrow$ & 1.20 \\
\hline NM_010514 & IGF2 & $\downarrow$ & 2.30 & $\downarrow$ & 1.90 \\
\hline
\end{tabular}

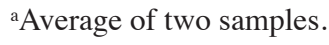

Gene expression profile of mouse Men1 knockout embryoid bodies in early pancreatic islet development. Since the homozygous Men1\% gene causes embryonic lethality to mice between embryonic days 11.5 and 13.5, changes in gene expression induced by Men1 knockout were examined during early development. Previous studies have indicated that embryoid bodies at day 8 of culture are equivalent to early organogenesisstage embryos (>8.5 d.p.c.) (14). Thus, mRNA isolated from the mouse Men $1 \%$ and Men1 ${ }^{+/+}$embryoid bodies on day 8 of culture were selected for microarray analysis. A substantial number of genes were up- or down-regulated in the Men1\% compared to the $\mathrm{Men}^{+/+}$embryoid bodies. When an arbitrary difference of $\geq 2$-fold change was selected, 517 transcripts were identified as down-regulated, including 377 known genes, and 70 transcripts were identified as up-regulated, including 41 known genes.

Among the genes examined, SOX4, FOXA1, BTC and IGF-2, which are involved in the development of pancreatic islets, were down-regulated in the Men $1 \%$ embryoid bodies (Table II).

Validation of changes in gene expression by quantitative $R T-P C R$. In order to validate the results of the microarray analysis, the mRNA expression levels of SOX4, FOXA1, BTC and IGF2 were analyzed by real-time quantitative RT-PCR. Two mRNA samples were used, including those used for the microarray. Although the fold changes in the expression of specific genes revealed by quantitative RT-PCR were not well correlated with those obtained by microarray analysis, the direction of change in the quantitative RT-PCR and microarray analysis was always concordant (Table II). The discrepancy between the fold changes can be explained by the very low expression levels in the sample (below the background level in the microarray analysis), leading to a fold change bias in the quantitative PCR analysis.

\section{Dicussion}

In a study reported by Bertolino et al (11), the generation of mice with a null mutation in the Men1 gene revealed a crucial role of this gene in embryonic development. However, due to its induction of early embryonic lethality in Men $1 \%$ mice, the authors were not able to address the role of menin in the development of endocrine tissues. Nevertheless, embryoid body formation has been shown to be a powerful in vitro model system for the study of early lineage determination and organogenesis in mammals, and such a system may serve as a useful tool for identifying the genes involved in development and differentiation $(14,15)$. In the present study, embryoid bodies were used as a model system to investigate the pathophysiological function of menin in pancreatic islet development and differentiation.

In previous studies, menin was demonstrated to be a key molecule for the suppression of the development of numerous types of endocrine tumors $(9,16)$. Menin was also shown to be required for the development of several organs and tissues, including the neural tube and liver. Of note, $\beta$-cell-specific Men1 mutant mice showed normal islet structures at an early age $(4,17)$. However, the possibility that this may be due to partial Cre-mediated gene disruptions among targeted cells during the embryonic stage cannot be excluded. Alternatively, menin may play its role before Rip-Cre recombinase (insulin) expression, similar to those genes whose function in pancreas development is required only during a narrow period. It was recently reported that menin is involved in the development of pancreatic endocrine cells, where it was found to be required for the formation of NEUROG3-expressing cells during the crucial step of the second pancreatic transition, in which the progenitor cells commit to endocrine differentiation (12).

In the present study, we found a difference in the time of gene expression between wild-type and mutant embryoid bodies for the SOX17 and FOXA2 genes, which are putative markers of definitive endoderm development. A detailed analysis of the expression of these genes during embryogenesis revealed an earlier phase of activity in the definitive endoderm of the postimplantation embryo. The definitive endoderm derives from the epiblast during gastrulation and, in the early organogenesis stage, forms the primitive gut tube, which gives rise to the digestive tract, liver, pancreas and associated visceral organs (18). Our results revealed that Men1\% embryonic stem cells may preferentially initiate differentiation along the endodermal pathway, as suggested by their early expression of FOXA2 and SOX17.

We also investigated the gene expression levels of certain genes involved in pancreatic islet development. Among the genes investigated, NKX2.2 was revealed to be downregulated on day 10 in the Men $1 \%$ embryoid bodies. NKX2.2 is a member of the mammalian NK2 homeobox transcription factor family, and is expressed in the ventral central nervous system and pancreas. NKX2.2 plays a unique role in endocrine pancreas development, and NKX2.2 null mice demonstrate a 
complete arrest of $\beta$-cell terminal differentiation, leading to the accumulation of a large group of cells that display endocrine characteristics and express a subset of $\beta$-cell markers, but lack insulin (18). Pancreatic $\beta$-cell-specific ablation of Men 1 mice leads to the progressive loss of insulin-positive staining in islet tumors. These results suggest a dedifferentiation process during insulinoma development in Men $1 \%$ mice, a phenomenon previously observed in a transgenic model of insulinoma (4). Moreover, up to $100 \%$ of Men 1 patients have been found to harbor small non-functioning pancreatic endocrine tumors in pathology studies (19). Taken together, the evidence indicates that the Men1 gene may act through the down-regulation of the genes involved in pancreatic islet differentiation, including NKX2.2, to induce the dedifferentiation of islet cells, which in turn leads to the tumorogenesis of islet. However, this proposed mechanism requires further investigation.

In our microarray study, we found a number of genes known to be implicated in pancreatic islet development to be down-regulated in Men1\% embryos, namely SOX4 (20), FOXA1, BTC and IGF2. Among these genes, we hypothesized that SOX4 might help elucidate the molecular events behind the process. Mice homozygous for a null mutation of the SOX4 gene failed to form normal islets, and NKX6.1, though not NKX.2.2, was significantly reduced in conjunction with the reduction in $\beta$-cells. This contradicts our other results, leading us to propose that another mechanism is probably involved.

In conclusion, we explored the expression of genes related to pancreatic islet development and differentiation in $\mathrm{Men1}^{-}$and $\mathrm{Men1}^{+/+}$embryoid bodies, in order to assess the pathophysiological function of menin in pancreatic islet development and differentiation. Our data indicate that Men1 may be involved in endoderm development, possibly through interaction with SOX17 or FOXA2, and may act through the down-regulation of NKX2.2 and SOX4 to affect pancreatic islet development. Collectively, the results of the present study suggest a critical role for Men1 in pancreatic islet development, and indicate that SOX17, FOXA2, NKX2.2 and SOX4 are potential targets of Men1.

\section{Acknowledgements}

This study was supported by the National Natural Sciences Foundation of China (30771021, 30800537). We are also grateful to Professor Zhang Chanxian and Dr Lu Jieli for their kind help in the research.

\section{References}

1. Pannett AA and Thakker RV: Multiple endocrine neoplasia type 1. Endocr Relat Cancer 6: 449-473, 1999.

2. Chandrasekharappa SC, Guru SC, Manickam P, et al: Positional cloning of the gene for multiple endocrine neoplasia-type 1. Science 276: 404-407, 1997.

3. Jensen RT: Pancreatic endocrine tumors: recent advances. Ann Oncol 10 (Suppl 4): 170-176, 1999.
4. Bertolino P, Tong WM, Herrera PL, Casse H, Zhang CX and Wang ZQ: Pancreatic beta-cell-specific ablation of the multiple endocrine neoplasia type 1 (MEN1) gene causes full penetrance of insulinoma development in mice. Cancer Res 63: 4836-4841, 2003.

5. Lemmens IH, Forsberg L, Pannett AA, et al: Menin interacts directly with the homeobox-containing protein Pem. Biochem Biophys Res Commun 286: 426-431, 2001.

6. Agarwal SK, Guru SC, Heppner C, et al: Menin interacts with the AP1 transcription factor JunD and represses JunD-activated transcription. Cell 96: 143-152, 1999.

7. Kaji H, Canaff L, Lebrun JJ, Goltzman D and Hendy GN: Inactivation of menin, a Smad3-interacting protein, blocks transforming growth factor type beta signaling. Proc Natl Acad Sci USA 98: 3837-3842, 2001.

8. Ohkura N, Kishi M, Tsukada T and Yamaguchi K: Menin, a gene product responsible for multiple endocrine neoplasia type 1, interacts with the putative tumor metastasis suppressor $\mathrm{nm} 23$. Biochem Biophys Res Commun 282: 1206-1210, 2001.

9. Heppner C, Bilimoria KY, Agarwal SK, et al: The tumor suppressor protein menin interacts with NF-kappaB proteins and inhibits NF-kappaB-mediated transactivation. Oncogene 20: 4917-4925, 2001

10. Sukhodolets KE, Hickman AB, Agarwal SK, et al: The 32-kilodalton subunit of replication protein $\mathrm{A}$ interacts with menin, the product of the MEN1 tumor suppressor gene. Mol Cell Biol 23: 493-509, 2003.

11. Bertolino P, Radovanovic I, Casse H, Aguzzi A, Wang ZQ and Zhang CX: Genetic ablation of the tumor suppressor menin causes lethality at mid-gestation with defects in multiple organs. Mech Dev 120: 549-560, 2003.

12. Fontaniere S, Duvillie B, Scharfmann R, Carreira C, Wang ZQ and Zhang CX: Tumour suppressor menin is essential for development of the pancreatic endocrine cells. J Endocrinol 199: 287-298, 2008

13. Stewart C, Parente F, Piehl F, et al: Characterization of the mouse Men1 gene and its expression during development. Oncogene 17: 2485-2493, 1998 .

14. Leahy A, Xiong JW, Kuhnert F and Stuhlmann H: Use of developmental marker genes to define temporal and spatial patterns of differentiation during embryoid body formation. J Exp Zool 284: 67-81, 1999

15. Novotny E, Compton S, Liu PP, Collins FS and Chandrasekharappa SC: In vitro hematopoietic differentiation of mouse embryonic stem cells requires the tumor suppressor menin and is mediated by Hoxa9. Mech Dev 126: 517-522, 2009.

16. Larsson C, Skogseid B, Oberg K, Nakamura Y and Nordenskjold M: Multiple endocrine neoplasia type 1 gene maps to chromosome 11 and is lost in insulinoma. Nature 332: 85-87, 1988.

17. Crabtree JS, Scacheri PC, Ward JM, et al: Of mice and MEN1: Insulinomas in a conditional mouse knockout. Mol Cell Biol 23: 6075-6085, 2003.

18. Zorn AM and Wells JM: Vertebrate endoderm development and organ formation. Annu Rev Cell Dev Biol 25: 221-251, 2009.

19. Radford DM, Ashley SW, Wells SA Jr and Gerhard DS: Loss of heterozygosity of markers on chromosome 11 in tumors from patients with multiple endocrine neoplasia syndrome type 1 . Cancer Res 50: 6529-6533, 1990.

20. Wilson ME, Yang KY, Kalousova A, et al: The HMG box transcription factor SOX4 contributes to the development of the endocrine pancreas. Diabetes 54: 3402-3409, 2005. 